Cahiers Charlevoix

Études franco-ontariennes
Cahiers Charlevoix Études franco-ontariennes or Crevereerie of:

\title{
Aspects de l'histoire des Franco-Ontariens du Centre et du Sud-Ouest, 1970-2000
}

\section{Yves Frenette}

Volume 10, 2014

URI : https://id.erudit.org/iderudit/1039293ar

DOI : https://doi.org/10.7202/1039293ar

Aller au sommaire du numéro

\section{Éditeur(s)}

Société Charlevoix

Presses de l’Université d'Ottawa

\section{ISSN}

1203-4371 (imprimé)

2371-6878 (numérique)

\section{Découvrir la revue}

\section{Citer cet article}

Frenette, Y. (2014). Aspects de l'histoire des Franco-Ontariens du Centre et du Sud-Ouest, 1970-2000. Cahiers Charlevoix, 10, 211-254.

https://doi.org/10.7202/1039293ar

\section{Résumé de l'article}

Yves Frenette continue à s'intéresser aux destinées des Franco-Ontariens du centre et du sud-ouest de la province. Il scrute les trois dernières décennies du $\mathrm{xx}^{\mathrm{e}}$ siècle, examinant, malgré la rareté des sources, certains aspects de l'évolution des Franco-Ontariens des deux régions. Grâce à un important apport migratoire, le nombre de francophones s'accroît dans le Centre, alors qu'il demeure stationnaire dans le Sud-Ouest. À l'orée du XxI ${ }^{\mathrm{e}}$ siècle, les deux régions abritent $35 \%$ des Franco-Ontariens de la province ; mais, comme la population totale de ces régions a aussi augmenté, le poids de cette francophonie demeure très faible. Néanmoins, ces Franco-Ontariens sont financièrement plus à l'aise et ils sont plus instruits que dans le reste de la province. Par ailleurs, dans les deux régions, les transferts linguistiques vers l'anglais continuent de plus belle, ce qui va de pair avec des transformations sur le plan de l'identité, notamment chez les jeunes. En raison de ces tendances et de l'éparpillement historique de la population de langue française, le réseau institutionnel est ténu et il fait face à de grands défis. Toutefois, le réseau scolaire prend de l'ampleur, notamment au palier secondaire, souvent après des luttes épiques. Après avoir vu le jour, les établissements doivent affronter de nombreux obstacles, le plus important étant leur petite taille. Quant aux programmes postsecondaires en français, ils sont très limités. Ce survol des deux régions permet à l'auteur de contraster l'évolution des communautés franco-ontariennes de Welland et de Toronto. Dans le premier cas, le déclin de la petite ville ouvrière se traduit en parallèle par celui de sa population de langue française, en dépit d'un certain essor institutionnel. À Toronto, par contre, la communauté francophone est dynamique et diversifiée, et traversée de redéfinitions identitaires et de questionnements institutionnels. En épilogue, Yves Frenette se demande si, en dépit du fractionnement qui ressort de son étude, une conscience régionale francophone ne serait pas en voie d'éclosion dans le Centre et le Sud-Ouest, en raison notamment de la fonction métropolitaine de Toronto et de la restructuration des conseils scolaires.
Ce document est protégé par la loi sur le droit d'auteur. L’utilisation des services d'Érudit (y compris la reproduction) est assujettie à sa politique d'utilisation que vous pouvez consulter en ligne.

https://apropos.erudit.org/fr/usagers/politique-dutilisation/ 


\title{
Aspects de l'histoire des Franco-Ontariens du Centre et du Sud-Ouest, 1970-2000
}

\author{
Yves Frenette
}

Chaire de recherche du Canada sur les migrations, les transferts et les communautés francophones

Université de Saint-Boniface 


\section{SOMMAIRE}

$\begin{array}{ll}\text { INTRODUCTION } & 213\end{array}$

I - $\quad$ Le Centre et le Sud-Ouest en tendances 214

II - TRANSFERTS Linguistiques ET tRAnSFormations IDENTITAIRES 218

III - LE PROBlÈME DES INSTITUTIONS 224

IV - Welland : LE DÉClin D'UNe COMMUNAUté FRANCO-ONTARIENNE 《TRADITIONNELLE 》 233

V - TORONTO : L'AVÈNEMENT D'UNE NOUVELLE FRANCOPHONIE 241

ÉPILOGUE 


\section{Aspects de l'histoire des Franco-Ontariens du Centre et du Sud-Ouest, 1970-2000}

\section{INTRODUCTION}

Dans une étude précédente parue dans les Cahiers Charlevoix, nous avons étudié l'évolution des régions du centre et du sudouest de 1'Ontario français entre 1940 et 1970. Nous avons alors fait ressortir que, contrairement à leurs compatriotes de l'est et du nord de la province, dont l'origine est en grande partie québécoise, les Franco-Ontariens du Centre et du Sud-Ouest ont des origines variées : française, canadienne, métisse, québécoise, acadienne, et que, en conséquence, leur identité a été plus fragmentée qu'ailleurs, en dépit d'un volumineux apport de migrants canadiensfrançais. Notre travail s'appuyait sur les recensements fédéraux, sur des études sociologiques effectuées pendant la décennie de 1960 ainsi que sur des témoignages et des enquêtes orales ${ }^{1}$.

Nous avons voulu effectuer le même exercice pour les trois dernières décennies du $\mathrm{Xx}^{\mathrm{e}}$ siècle, c'est-à-dire rendre compte de l'évolution de l'Ontario français du Centre et du Sud-Ouest en faisant appel à une kyrielle de sources. Mais celles-ci se sont avérées difficiles à cerner pour l'élaboration d'une synthèse. Les recensements fédéraux devenant de plus en plus sophistiqués à partir de 1971, il aurait été possible d'entreprendre une étude exhaustive de la population franco-ontarienne des deux régions, mais tel n'était pas notre but ${ }^{2}$. Nous avons donc utilisé cette source

1. Yves Frenette, «L'Ontario français du Centre et du Sud-Ouest, 1940-1970 », dans Cahiers Charlevoix, $\mathrm{n}^{\circ}$ 7, Sudbury, Société Charlevoix et Prise de Parole, 2006, p. 145-183. Nous reprenons les définitions des frontières régionales que nous donnions alors, définitions qui sont empruntées à l'Office des affaires francophones de l'Ontario : le Centre s'étend de la limite sud de Trenton jusqu'à Fort-Erié, englobant la Baie-Georgienne ; le Sud-Ouest, quant à lui, va de l'agglomération de London jusqu'à la frontière avec le Michigan. Voir Office des Affaires francophones, Profil statistique. Les francophones en Ontario, Toronto, Office des Affaires francophones, s. d., p. 1.

2. Nous avons tenté l'expérience il y a une dizaine d'années dans « Les Franco- 
avec retenue. Quant aux entrevues, elles sont rares. Il a donc fallu faire montre d'imagination et recourir à des documents peu exploités par les historiens, par exemple des bulletins d'association et des bottins spécialisés. Nous avons aussi puisé abondamment dans les travaux d'autres chercheurs.

Toutefois, nous n'avons pas obtenu le résultat souhaité et, au lieu d'une synthèse, nous proposons au lecteur une étude de certains aspects de l'histoire des Franco-Ontariens du Centre et du Sud-Ouest entre 1970 et 2000. Ainsi, dans un premier temps, nous traçons la progression numérique de la population de langue française et les grandes tendances de l'évolution de ce groupe minoritaire. Nous observons ensuite les transferts linguistiques et les transformations identitaires ayant eu cours dans les deux régions, avant de nous pencher sur les problèmes auxquels ont dû faire face les institutions franco-ontariennes. Nous terminons notre esquisse en contrastant le déclin de la francophonie de Welland, petite communauté considérée par plusieurs comme le château-fort franco-ontarien du centre de l'Ontario, et l'avènement d'une communauté francophone dynamique mais éclatée dans la métropole torontoise.

\section{I - Le Centre et le Sud-Ouest en tendances}

En 1971, le Centre et le Sud-Ouest regroupent 146360 personnes ayant déclaré au recenseur qu'elles étaient de langue maternelle française ${ }^{3}$. À ce moment, il n'est malheureusement pas possible de distinguer la population franco-ontarienne du Centre et du Sud-Ouest, qui sont amalgamés dans la grande région du « Sud ». Il faut attendre le recensement de 1976 pour cela (tableau 1). Comme on peut le constater, à cette date, les francophones du Centre sont presque trois fois plus nombreux que ceux du Sud-

phones du centre et du sud-ouest de l'Ontario. Un portrait sommaire », dans Marcel Bénéteau (dir.), Le Passage du Détroit. 300 ans de présence francophone-Passages. Three Centuries of Francophone Presence at Le Détroit, Windsor, Humanities Research Group, 2003, p. 289-297.

3. À moins d'indication contraire, les statistiques présentées dans cette étude proviennent des recensements fédéraux de la population. 
Ouest ; toutefois, leur pourcentage de la population totale est plus bas, $1,7 \%$ comparé à $2,9 \%$. Ce faible poids démographique se retrouve à l'échelle locale, à deux exceptions, Welland (16,1\%) dans le Centre et Windsor (5,9\%) dans le Sud-Ouest.

\section{Tableau 1}

La population franco-ontarienne du Centre et du Sud-Ouest, 1976

\begin{tabular}{|l|c|c|c|c|}
\hline & $\begin{array}{c}\text { Population } \\
\text { totale }\end{array}$ & $\begin{array}{c}\text { Langue } \\
\text { maternelle } \\
\text { française }\end{array}$ & $\begin{array}{c}\% \text { de } \\
\text { population } \\
\text { francophone } \\
\text { de la région }\end{array}$ & $\begin{array}{c}\% \text { de } \\
\text { population } \\
\text { francophone } \\
\text { de la province }\end{array}$ \\
\hline $\begin{array}{l}\text { Centre et } \\
\text { Sud-Ouest }\end{array}$ & 8264350 & 123930 & 2,0 & 26,8 \\
\hline Centre & 5050830 & 87245 & 1,7 & 18,9 \\
\hline Sud-Ouest & 1247140 & 36685 & 2,9 & 7,9 \\
\hline
\end{tabular}

Pendant le quart de siècle suivant, la population de langue maternelle française du Centre et du Sud-Ouest augmente ses effectifs de $60 \%$ pour atteindre 198695 personnes en 2001. Cette croissance fulgurante est due essentiellement aux migrations vers le Centre, puisque la population francophone du Sud-Ouest ne croît pas et que, par ailleurs, les transferts linguistiques vers l'anglais prennent beaucoup d'ampleur, comme nous le verrons ci-dessous. Au tournant du XxI ${ }^{\mathrm{e}}$ siècle, les francophones des deux régions représentent $35 \%$ de la population franco-ontarienne, comparativement à $27 \%$ en 1976. Mais, comme la population totale du Centre et du Sud-Ouest a aussi augmenté, particulièrement dans la première région, le poids démographique des francophones demeure très faible.

\section{Tableau 2}

La population franco-ontarienne du Centre et du Sud-Ouest, 2001

\begin{tabular}{|l|c|c|c|c|c|}
\hline & $\begin{array}{c}\text { Population } \\
\text { totale }\end{array}$ & $\begin{array}{c}\text { Langue } \\
\text { maternelle } \\
\text { française }\end{array}$ & $\begin{array}{c}\% \\
\text { évolution } \\
\text { depuis 1976 }\end{array}$ & $\begin{array}{c}\% \text { de } \\
\text { population } \\
\text { franco-ontarienne } \\
\text { de la région }\end{array}$ & $\begin{array}{c}\% \text { de } \\
\text { population } \\
\text { franco-ontarienne } \\
\text { de la province }\end{array}$ \\
\hline $\begin{array}{l}\text { Centre et } \\
\text { Sud-Ouest }\end{array}$ & 9672965 & 198695 & $+60,3$ & 2,0 & 34,6 \\
\hline Centre & 8158825 & 162070 & $+85,8$ & 2,0 & 28,7 \\
\hline Sud-Ouest & 1514140 & 36625 & $-0,2$ & 2,3 & 5,9 \\
\hline
\end{tabular}


Nous avons affaire à des individus ayant complété leurs études secondaires dans une proportion de $82,1 \%$ dans le Centre et de $77,2 \%$ dans le Sud-Ouest, des taux de scolarité qui, dans les deux cas, dépassent de $2 \%$ ceux de la population totale. Cependant, l'écart entre les deux régions est plus grand pour les FrancoOntariens ayant fait des études postsecondaires, respectivement $51,7 \%$ et $39,5 \%$, pourcentages qui correspondent aux taux de la population totale. Il y a sans doute une corrélation entre ces niveaux de scolarité et le revenu familial moyen qui est de 80749 \$ dans le Centre et de 66105 \$ dans le Sud-Ouest, comparé à $70033 \$$ pour l'ensemble de l'Ontario français. Dans la première région, le revenu familial moyen des Franco-Ontariens est de plus de $4000 \$$ supérieur à celui de la population totale et dans la seconde, il se situe en-deçà de 2000 \$à celui de la population totale. La pauvreté est le lot de $14 \%$ des Franco-Ontariens dans les deux régions. Ici, il n'y a pas d'écart avec la population totale.

La croissance économique du Centre et ses besoins en maind'œuvre ainsi que la demande de personnel bilingue à Queen's Park, dans les entreprises et dans certains établissements scolaires contribuent à attirer de nombreux francophones dans la région. La migration des cerveaux en provenance du Nord, l'accueil des réfugiés politiques et l'arrivée de migrants économiques originaires des pays en voie de développement ajoutent à cette souche et la renforcent. La présence française augmente particulièrement dans les cantons de Halton, York et Peel, faisant du grand Toronto le milieu qui change le plus eu égard au nombre de FrancoOntariens à l'échelle de la province. London et ses environs voient presque tripler leur population de langue maternelle française. Le nombre de francophones se multiplie aussi par trois dans Kitchener-Waterloo. Ainsi, l'espace géographique francophone s'étend-il. Mais, comme le remarque avec justesse Anne Gilbert, ces cellules de vie française ne constituent pas des maillons forts du territoire, puisqu'elles ne comptent généralement que quelques milliers de francophones dispersés à l'échelle des comtés. Les pertes parfois lourdes subies par certaines composantes tradition- 
nelles de l'espace franco-ontarien ont des effets marqués. En dépit de la Loi sur les services en français en 1988, cette langue reste quasiment invisible dans la région. L'importance relative des francophones y est si négligeable que les possibilités concrètes de vie française demeurent extrêmement limitées et, à l'exception de Toronto, le développement institutionnel s'y effectue difficilement ${ }^{4}$.

La situation à la Baie-Georgienne au tournant du $\mathrm{XXI}^{\mathrm{e}}$ siècle illustre les deux faces de Janus des communautés francophones du Centre. D’une part, la population de Pénétanguishene et de ses alentours, population dont les origines remontent à 1830 , est vieillissante et les perspectives d'avenir sont axées sur le développement communautaire, telle la création d'infrastructures adaptées aux loisirs d'une population qui est en préretraite ou les soins domiciliaires et de santé. À l'exception des secteurs de la construction résidentielle et du service téléphonique, l'activité économique demeure concentrée autour des institutions sociales, politiques et économiques déjà en place. Il existe toutefois des domaines où l'expansion est considérable ${ }^{5}$. À bien des égards, le nord de la Baie-Georgienne ressemble aux vieilles communautés d'Essex et de Kent, dans le Sud-Ouest' ${ }^{6}$.

D'autre part, la ville de Barrie connaît une des croissances démographiques les plus spectaculaires au Canada. Les habitants sont plus jeunes que dans la partie nord de la Baie-Georgienne et le tiers y sont établis depuis moins de cinq ans. La population est composée en partie de navetteurs qui se rendent quotidiennement à Toronto, où ils travaillent surtout comme employés de bureau et ouvriers de la construction. Sur place, les possibilités d'emplois pour les personnes bilingues se trouvent dans le commerce de

4. Anne Gilbert, Espaces franco-ontariens, Hearst, Le Nordir, 1999, p. 48-52.

5. Normand Labrie et al., " Mondialisation et exploitation des ressources linguistiques. Les défis des communautés francophones de l'Ontario », The Canadian Modern Language Review - La revue canadienne des langues vivantes, vol. 57, $\mathrm{n}^{\circ} 1$ (septembre 2000), p. 94-106.

6. Sur les francophones du Sud-Ouest à la fin du $\mathrm{Xx}^{\mathrm{e}}$ siècle, voir Gilbert, op. cit., p. $48-49$. 
proximité et dans certaines entreprises qui visent le marché canadien ${ }^{7}$.

La population francophone du Centre accroît encore sa diversité dans les trois dernières décennies du $\mathrm{Xx}^{\mathrm{e}}$ siècle. Au recensement de 2001, alors que presque les deux tiers des francophones de l'Ontario sont nés dans la province, comme c'est le cas dans la région voisine du Sud-Ouest, ce taux baisse à $42 \%$ dans le Centre. De toutes les régions ontariennes, c'est elle qui compte la plus forte proportion de Québécois $(30,7 \%)$ et de francophones nés à l'extérieur du Canada (16,7\%) (tableau 3). En fait, plus de la moitié $(55,2 \%)$ des immigrants francophones de l'Ontario vit dans le Centre. Quant au Sud-Ouest, il ne compte que 2780 francophones membres d'une minorité visible, soit 8,5\% de la population francophone régionale et $4,8 \%$ des minorités raciales francophones de l'Ontario.

Tableau 3

Lieux de naissance des francophones du Centre et du Sud-Ouest, 2001 (\%)

\begin{tabular}{|l|c|c|c|c|}
\hline $\begin{array}{l}\text { Lieu de } \\
\text { naissance }\end{array}$ & Ontario & Québec & $\begin{array}{c}\text { Autres } \\
\text { provinces }\end{array}$ & $\begin{array}{c}\text { Extérieur } \\
\text { du Canada }\end{array}$ \\
\hline Ontario & 65,1 & 22,7 & 5,4 & 6,8 \\
\hline Centre & 42,2 & 30,7 & 10,4 & 16,7 \\
\hline Sud-Ouest & 63,9 & 20,7 & 8,4 & 7,0 \\
\hline
\end{tabular}

\section{II - TrANSFERTS LINGUISTIQUES ET TRANSFORMATIONS IDENTITAIRES}

En 1970, cela fait belle lurette que les transferts linguistiques vers l'anglais sévissent chez les Franco-Ontariens du Centre et du Sud-Ouest ${ }^{8}$. En effet, depuis la Deuxième Guerre mondiale, le ratio langue maternelle française/origine ethnique française a constamment décliné pour atteindre $50 \%$. À partir de ce moment, les observateurs de la scène francophone peuvent utiliser une mesure plus fiable du taux de conservation linguistique, soit le ratio langue parlée à la maison/langue maternelle. Celui-ci se situe alors à $43,5 \%$ et se maintient plus ou moins jusqu'au

7. Labrie, loc. cit.

8. Frenette, op. cit., p. 165-168. 
milieu des années 1990, alors qu'il décline au tiers. Avec un taux de $34,3 \%$, le Centre se tire à peine mieux d'affaire que la vieille région francophone du Sud-Ouest, où seulement 29,6 \% des personnes de langue maternelle française parlent couramment français à la maison.

Au tournant du XXI ${ }^{\mathrm{e}}$ siècle, la plupart des anciennes paroisses de langue française du diocèse de London sont passées à l'anglais. En effet, dans le Sud-Ouest, le taux de conservation linguistique a encore décliné pour atteindre 26,9\%. Dans toute la province, la région est celle où l'on observe la plus forte baisse du taux de conservation du français chez les jeunes (24 ans et moins) depuis 1996. La situation est meilleure dans le Centre, où le tiers des Franco-Ontariens utilisent surtout le français à la maison. Ces pourcentages sont bien inférieurs à celui de la francophonie ontarienne dans son ensemble, qui est de 56,5\%. Comme l'ont montré plusieurs chercheurs, notamment le regretté Roger Bernard, les transferts linguistiques sont étroitement liés au phénomène des mariages mixtes 9 . Sans surprise, on constate que ce phénomène domine dans le Centre et le Sud-Ouest, le pourcentage de ménages exogames étant respectivement de $81,5 \%$ et de $82,2 \%$.

Dans les écoles, la majorité des élèves provient de foyers mixtes ou anglophones. La situation est particulièrement inquiétante dans les établissements d'enseignement secondaire, dans lesquels l'anglais domine partout à l'extérieur de la salle de classe. À certains endroits, les élèves s'adressent parfois même aux enseignants et à la direction dans cette langue. Certains enseignants vont jusqu'à utiliser des mots anglais pour établir un rapport avec la classe : « Vous n'avez pas de devoirs au mois de juin if (et il y a toujours un if) vous faites parfaitement d'ici au 31 mai. Is it

9. Sur l'exogamie et ses conséquences en milieu francophone à l'extérieur du Québec, voir Roger Bernard : « Langue maternelle et langue d'usage dans les foyers mixtes. Les enjeux de l'exogamie », dans Cahiers Charlevoix, $\mathrm{n}^{\circ} 1$, Sudbury, Société Charlevoix et Prise de Parole, 1995, p. 241-289 ; "Transferts linguistiques et anglicisation des francophones. Les enjeux de l'exogamie au Canada », dans Cahiers Charlevoix, $\mathrm{n}^{\circ}$ 2, Sudbury, Société Charlevoix et Prise de Parole, 1997, p. 232-247; "Langue d'usage avec les parents à l'intérieur des foyers mixtes francophones. Les enjeux de l'exogamie », dans Cahiers Charlevoix, $\mathrm{n}^{\circ} 4$, Sudbury, Société Charlevoix et Prise de Parole, 2000, p. 13-49. 
a deal ? Marché conclu ${ }^{10}$. » À Hamilton, London et Sarnia, les jeunes ont parfois honte de parler français dans la rue, car on se moque d'eux ou « on les trouve bizarres ». Les élèves exagèrent peut-être l'antipathie des anglophones, mais c'est leur perception négative de la langue et de la culture françaises qui importe. Ils perçoivent généralement le fait français comme vieillot; qui plus est, ils semblent interpréter les efforts des directions d'école et du personnel pour les conscientiser comme une imposition qui, en fait, les éloigne, plutôt que de les rapprocher de la culture francoontarienne. Il est significatif que la Fédération de la jeunesse franco-ontarienne (dorénavant FESFO) soit peu présente dans les écoles du Centre et du Sud-Ouest et qu'elle suscite peu d'intérêt. Lors d'une enquête dans certains établissements secondaires du secteur public, l'impression qui s'est dégagée presque partout est celle d'une dissociation de la langue et de la culture françaises. Le problème est aggravé par la présence dans les établissements scolaires d'une proportion parfois importante d'élèves provenant des écoles élémentaires d'immersion. Pour eux, le français ne représente qu'un outil de communication ${ }^{11}$.

Toutefois, les contextes locaux sont déterminants. Plus l'environnement est francophone, plus les jeunes ont tendance à s'exprimer en français et à maîtriser cette langue. À l'école Étienne-Brûlé de Toronto et, à un degré moindre, à l'école secon-

10. Monica Heller, « Variation dans l'emploi du français et de l'anglais par les élèves des écoles de langue française de Toronto ", dans Raymond Mougeon et Édouard Béniak (dir.), Le Français parlé hors Québec. Aperçu sociologique, Québec, Presses de l'Université Laval, 1989, p. 165.

11. Cette section sur les élèves des écoles secondaires du Centre et du SudOuest s'appuie sur une enquête réalisée par Maurice Lamontagne, Yves Frenette et Pierre Bourgeois, Rapprochement école - communauté. Une réflexion sur l'avenir des communautés et des écoles publiques de langue française du Centre-Sud-Ouest, Toronto, Conseil scolaire de district du Centre-Sud-Ouest, 2000, 31 p. L'enquête fut commanditée par ce conseil, qui s'inquiétait alors de la perte d'effectifs lors du passage entre les différents paliers scolaires et de la concurrence des conseils scolaires de langue anglaise. Pour réaliser cette étude, nous avons étudié les chiffres du recensement fédéral de 1996 et certains documents internes du conseil. Surtout, nous avons effectué des visites de chaque école secondaire et nous avons recueilli les témoignages des directions, des enseignants et des élèves. Comme son titre l'indique, le rapport préconisait un rapprochement entre les établissements et leurs communautés respectives ainsi que des stratégies pour y arriver. 
daire Le Caron de Pénétanguishene, la langue française semble ainsi être associée à une culture française. Dans le premier cas, cette culture varie selon l'origine ethnoculturelle des élèves ; à Le Caron, la culture est canadienne-française, même si la majorité des adolescents insistent, comme ailleurs, sur le fait qu'ils sont « des bilingues » : ils reconnaissent la distinction simple entre « les Français » et « les Anglais » et s'identifient aux premiers. Cette identification semble reposer tout autant sur leur capacité à s'exprimer en français que sur la reconnaissance de leur héritage culturel. En effet, tous les élèves de la Baie-Georgienne que rencontre le politologue François-Pierre Gingras au début de la décennie de 1990 se disent « français » ou « canadiens-français », même ceux qui s'expriment avec une certaine difficulté dans la langue de Molière. Ils semblent être influencés par des confrères et consœurs dont la maîtrise du français se situe au-dessus de la moyenne et qui proviennent de foyers où on parle surtout cette langue. Ces individus sont peu nombreux, mais ils jouent en quelque sorte le rôle de « conscientiseurs » de leurs camarades en militant pour la « cause franco-ontarienne ». Utilisant le plus possible le français dans leur vie quotidienne, ils essaient de fréquenter des amis qui n'ont pas peur de s'exprimer dans cette langue et ils participent à des activités qui se déroulent entièrement en français, que ce soit dans les arts, les sports ou des associations de jeunes ${ }^{12}$.

Ailleurs, des représentations négatives du français parlé localement créent l'insécurité linguistique et contribuent subtilement aux transferts vers l'anglais. Le canton de Dover, dans le sudouest de la province, est exemplaire à cet égard. Dans la dernière décennie du $\mathrm{Xx}^{\mathrm{e}}$ siècle, on y trouve quatre écoles élémentaires de

12. François-Pierre Gingras, «Identité : jeune, francophone minoritaire en Ontario ", Francophonies d'Amérique, no 3 (1993), p. 91-99. Depuis vingt ans, le sociologue Simon Laflamme a fait paraître de nombreux travaux sur l'alternance linguistique et le rapport aux médias des Franco-Ontariens. Dans cette abondante production, contentons-nous de deux exemples : Simon Laflamme et Christiane Bernier, Vivre dans l'alternance linguistique. Médias, langue et littératie en Ontario français, Sudbury, Centre Fora, 1998, 124 p. ; Simon Laflamme, «Les Médias en Ontario chez les francophones et les anglophones », Cahiers Charlevoix, $\mathrm{n}^{\circ}$ 6, Sudbury, Société Charlevoix et Prise de Parole, 2004, p. 249-282. 
langue française, qui comptent environ 900 élèves, et une école secondaire de langue française d'environ 300 élèves. Le taux de continuité scolaire entre les deux paliers est très élevé $(90 \%)$ et le taux de décrochage au secondaire est minime (moins de $5 \%$ ). Quoique le français standard soit transmis à l'école, dans les médias et à l'église, le français local, caractérisé par la diglossie, l'emprunt de mots anglais francisés et l'utilisation d'archaïsmes transmis par les générations précédentes, dominent. Fière d'être bilingue, la population de langue française souffre d'insécurité linguistique ; plusieurs personnes qui ont fait leurs études secondaires en anglais, faute d'établissement de langue française avant la décennie de 1970, décrivent leur langue comme un «franglais $\gg^{13}$. Cependant, chez les plus jeunes, le bilinguisme est de plus en plus valorisé, surtout chez ceux œuvrant dans les secteurs des technologies de l'information et des communications ainsi que dans quelques industries locales qui ont établi des marchés à l'échelle nationale ou internationale. Ayant l'occasion d'utiliser le français dans leur espace professionnel, surtout dans des postes qui relèvent du service à la clientèle, ces jeunes adultes reconnaissent que le bilinguisme représente un atout. Cette prise de conscience ne s'étend toutefois pas aux autres espaces de leur vie, notamment la famille ${ }^{14}$.

Il existe des milliers d'exemples de Franco-Ontariens du Centre et du Sud-Ouest qui sont devenus bilingues ou complètement anglophones. Beaucoup plus rares sont les personnes qui, très jeunes, développent un fort sentiment d'appartenance à la culture franco-ontarienne. En dépit de leur petit nombre, ils sont très importants dans la francophonie régionale parce que ce sont eux qui s'engagent sur le plan communautaire et en viennent souvent à jouer un rôle de leadership. Par exemple, Peter Hominuk naît à Welland en 1968, où il passe sa jeunesse, un peu à l'extérieur du « quartier social » ou Frenchtown. Son père étant d'origine

13. Roger Lozon, « Les Variétés de langue parlées dans le canton de Dover. Sources de fierté ou sentiments d'insécurité ? », dans Marcel Bénéteau, op. cit., p. 289.

14. Id., « Les Jeunes du Sud-Ouest ontarien. Représentations et sentiments linguistiques ", Francophonies d'Amérique, no 12 (2001), p. 83-92. 
ukrainienne et sa mère franco-ontarienne, il apprend l'anglais et le français en même temps. Il fait toutes ses études en français dans le système public et il n'a jamais envie de transférer dans une école anglaise : «Pour moi, dit-il, c’était quelque chose de très important de continuer en français [...] Il n'y a jamais eu de question pour moi : je m'en allais à l'école Confédération au secondaire, pis ça finissait là. » Comme les autres élèves, Peter parle français dans la salle de classe et anglais dans les corridors. Très impliqué dans les activités parascolaires, il devient membre de l'Association canadienne-française de l'Ontario (dorénavant ACFO) régionale du Niagara. C'est lorsqu'il entre en contact avec des jeunes d'autres régions qu'il prend conscience de l'isolement de la communauté francophone de Welland:

[...] j'ai senti des manques. Les gens me parlaient d'artistes franco-ontariens, j'avais vraiment aucune idée de qui ils parlaient. On entendait souvent parler, à Radio-Canada, de certaines personnes, mais passé ça, c'est un manque dans la communauté du Sud. Je me rappelle, au secondaire, de temps en temps, il y avait des spectacles francophones que l'école faisait venir. On a réalisé que du rock, ça pouvait être francophone pis pas être plate. Ça été une grosse surprise pour nous au secondaire.

Après ses études secondaires, Hominuk entre à l'emploi de l'Association de l'accueil jeunesse de l'Ontario, puis il occupe divers postes à l'Association des gens de la presse de l'Ontario, à 1'AcFo régionale du Niagara, à Direction-Jeunesse, à la Télévision française de l'Ontario et à la Clef de la Baie. Au moment d'écrire ces lignes, il occupe le poste de directeur général de l'Assemblée franco-ontarienne ${ }^{15}$.

Parfois, des francophones qui se sont éloignés de leur langue et de leur culture les retrouvent plus tard dans la vie. Natif de Lafontaine, à la Baie-Georgienne, Basile Dorion a vécu pendant dix ans en anglais à Toronto et à Midland.

15. Entrevue de Peter Hominuk réalisée par Mary-Elizabeth Aubé, Toronto, 8 juin 1998. Pour un autre exemple d'une jeune francophone conscientisée, voir Brian Tanguay, "Neither Dead Ducks nor Warm Corpses. The Francophones of Dover Township (Kent County) », dans Bénéteau, op. cit., p. 334. 
Alors, à un moment donné, ça m'a frappé que ce que j'avais de très précieux en moi, qui était une langue et une culture de langue française, j'étais en train de m'en éloigner. J'allais perdre quelque chose que j'imaginais qui ne pouvait pas se perdre... Alors l'importance que ma langue et ma culture soient transmises à l'autre génération qui venait après moi, c'est devenu très important à ce stage-là ${ }^{16}$.

En 1978, Dorion devient directeur du Centre d'activités françaises de Pénétanguishene. Conseiller scolaire, il est un des leaders dans la lutte pour l'obtention d'une école secondaire de langue française, même s'il se présente originellement pour contrer les éléments franco-ontariens qu'il considère radicaux : « je les trouvais des fous de se battre si fort pour la francophonie. Trois mois plus tard, c'est eux autres qui me retenaient ${ }^{17} \gg$.

\section{III - LE PROBLÈME DES INSTITUTIONS}

Comme avant 1970, l'éparpillement de la population francoontarienne a un impact important sur la vie française dans le Centre et le Sud-Ouest. Le réseau institutionnel est plus ténu qu'ailleurs en province et il répond surtout aux besoins des gens de 50 ans et plus. Pour une grande partie des francophones, les activités communautaires reflètent la culture de la majorité anglophone. Toutefois, avec l'expansion du réseau scolaire, l'arrivée d'enseignants de langue française du nord et de l'est de la province ainsi que du Québec engendre un dynamisme nouveau. Puis, en 1988, la mise en œuvre de la Loi 8 représente une pierre blanche dans l'évolution des services en français, puisque la majorité des milieux urbains des deux régions sont désignés bilingues.

Par ailleurs, la croissance de la population de langue française ne se traduit pas par la création de nouvelles paroisses après 1970. On n'en fonde que deux, Sainte-Famille et Saint-Martyrs canadiens, qui voient le jour à Brampton en $1976^{18}$.

16. « Basile Dorion », dans François-Xavier Chamberland, L'Ontario français se raconte. De $A$ à X, Toronto, Éditions du Gref, 1999, p. 538.

17. Ibid., p. 547.

18. Gaétan Gervais, « Les Paroisses de l’Ontario français 1767-2000 », dans 
À l'extérieur de Toronto, c'est le club social qui continue à être l'équipement culturel le plus répandu chez les Franco-Ontariens du Centre et du Sud-Ouest. En 1977, lorsque l'historien Pierre Savard, mandaté par le Conseil des arts de l'Ontario, effectue une tournée dans les deux régions, on en compte une trentaine. Ils se sont développés à partir du besoin des Franco-Ontariens de se récréer en français. Une activité sportive, par exemple les quilles, en fournit l'occasion : on met sur pied une ligue et, à la fin de la saison, on fête les champions lors d'un banquet et d'une soirée de danse; puis un club social se forme et se dote d'une structure capable d'organiser des activités sportives, sociales, culturelles et artistiques ; le nombre de membres s'accroît. Le club type sert au besoin de groupes de pression, souvent de concert avec les conseils régionaux de l'Association canadienne-française de l'Ontario, pour revendiquer une école, la radio ou la télévision françaises, ou encore des services bilingues. Aux danses viennent s'ajouter des spectacles musicaux et théâtraux, ainsi que des cours et ateliers dans divers médias artistiques. Parfois, un centre culturel naît. Le club social peut aussi ouvrir un comptoir où les francophones peuvent se procurer livres et disques français. Enfin, il organise à l'occasion une garderie et des programmes pour les enfants : camps d'été, terrains de jeux, etc. ${ }^{19}$

Les clubs sociaux du Centre et du Sud-Ouest sont regroupés dans la Fédération des clubs sociaux franco-ontariens, fondée en 1959, et comptant environ 8000 membres quelque vingt ans plus tard. La Fédération vise à accroître et à faciliter les contacts entre membres. Elle organise des tournois sportifs et des activités socioculturelles. Elle publie aussi un bulletin mensuel d'information, Le Trait d'union. Ses clubs membres forment un réseau utile à l'organisation de tournées de spectacles, tout en encourageant la création de chorales et de groupes folkloriques. Les manifestations culturelles qui se déroulent en français sont

Cahiers Charlevoix, $\mathrm{n}^{\circ}$ 6, Sudbury, Prise de Parole et Société Charlevoix, 2004, p. 172.

19. Pierre Savard, Cultiver sa différence. Rapport sur les arts dans la vie francoontarienne, Toronto, Conseil des arts de l'Ontario, 1977, p. 90-96. 
souvent inspirées par le passé. Soirées « canadiennes du bon vieux temps », cafés-chantant, chansonniers et folkloristes sont en vogue parmi les gens d'âge mûr. On peut ainsi parler d'une « culture de souvenir », qui fait renaître les activités culturelles typiques des lieux d'origine des participants ${ }^{20}$. Ne répondant plus aux besoins des francophones, la Fédération est dissoute au milieu des années 1990.

Au fil des ans, les communautés de langue française des deux régions s'étaient dotées d'un réseau impressionnant d'écoles élémentaires. En 1969, on comptait ainsi 12853 élèves dans 48 établissements $^{21}$. À partir de ce moment, il est très difficile de chiffrer la progression du réseau scolaire de langue française. Nous devons, pour l'instant, nous contenter d'une esquisse.

Au début des années 1970, la création de comités consultatifs de langue française (dorénavant CCLF) au sein de plusieurs conseils scolaires a comme résultat l'ouverture d'écoles élémentaires de langue française partout sur le territoire du Centre et du Sud-Ouest. Toutefois, les mentalités ne se transforment que lentement et, en 1977, le réseau scolaire de langue française n'atteint qu'une petite fraction des jeunes ${ }^{22}$. Dans les deux décennies suivantes, dans la foulée de l'avènement de la Charte des droits et libertés en 1982 et par l'action de groupes de parents, on assiste à la mise sur pied de nombreuses écoles confessionnelles et publiques de langue française. Ce ne sera qu'en 1998 que les FrancoOntariens obtiendront la gestion de leurs institutions scolaires. Dans le Centre et le Sud-Ouest, ils bénéficieront ainsi des services de deux conseils scolaires confessionnels et d'un conseil scolaire public.

$\mathrm{Au}$ palier secondaire, avant 1968, il existait dans les deux régions quelques établissements ou sections de langue française. Dès l'entrée en vigueur des lois 140 et 141, des écoles publiques

20. Ibid.

21. Comité franco-ontarien d'enquête culturelle, La Vie culturelle des FrancoOntariens, Ottawa, 1969, p. 113.

22. Savard, op. cit., p. 94. 
de langue française voient le jour à Paincourt, à Welland et à Toronto. Ailleurs, ce n'est qu'après une âpre lutte que les francophones auront gain de cause. Ainsi, dès l'adoption des deux lois, des militants de Windsor-Essex, appuyés par 1'ACFO, réclament une école secondaire publique de langue française. Il leur faudra plus de dix ans pour l'obtenir en raison de l'opposition des conseils scolaires d'Essex et de Windsor, la concurrence entre ces deux entités ainsi que le lobby de la «Essex County Ratepayers Association », fondée en janvier 1976. Après plusieurs vaines tentatives de médiation, le gouvernement conservateur de Bill Davis se résoudra, 1'année suivante, à présenter une loi : «Bill 3 - An Act to Require the Essex County Board of Education to provide a French Language Secondary School ». L'école secondaire L'Essor ouvre finalement ses portes et reçoit ses 450 premiers élèves en septembre $1979^{23}$.

Au même moment, à l'école secondaire de Pénétaguishene-, un établissement soi-disant bilingue qui a ouvert ses portes en 1969 et où les cours et services en français sont offerts au compte-gouttes -, une élève de $13^{\mathrm{e}}$ année, Denise Jaiko, rédige une proposition pour créer une véritable école bilingue. Le document est entériné par le CCLF, mais le conseil scolaire ne bouge presque pas. Le 15 mai, a lieu une manifestation à Queen's Park ; le ministre de l'Éducation, Beth Stephenson, hésite à intervenir. C'est alors qu'un groupe de Franco-Ontariens, qui est appuyé par 1'ACFO, l'Association des enseignants et des enseignantes francoontariens ainsi que par le chef de l'opposition à la Chambre des communes, Pierre Elliott Trudeau, met sur pied une école de langue française parallèle dans les locaux d'un centre culturel. Le 3 septembre 1979, après deux jours de festivités, s'ouvre ainsi la $33^{\mathrm{e}}$ école secondaire de langue française de l'Ontario, un établissement illégal, né de la résistance. Malgré de nombreux dons et appuis financiers, les ressources pédagogiques y demeurent fragmentaires. Devant l'opposition de la Ville de Pénétan-

23. René Godin et Lise Roy-Poirier, Windsor, Ottawa, Centre franco-ontarien de ressources pédagogiques, 1982, p. 145-161. 
guishene, qui demande une injonction pour fermer l'école en vertu d'un règlement de zonage, et l'inaction de Queen's Park, les militants, qui continuent à recevoir des appuis provinciaux et nationaux, créent un conseil scolaire parallèle. Finalement, en avril 1980, à la veille du référendum sur la souveraineté du Québec, le gouvernement Davis annonce la construction d'une école secondaire régionale de langue française. Elle prend le nom de Le Caron ${ }^{24}$. Une fois de plus, le conflit a fait ressortir les divisions au sein de la population franco-ontarienne de la BaieGeorgienne : les querelles familiales sont fréquentes et des amitiés de longue date sont parfois brisées ${ }^{25}$.

Après leur mise sur pied, les écoles secondaires de langue française du Centre et du Sud-Ouest font face à de nombreux défis, dont le plus important est sans doute leur petite taille ${ }^{26}$. Parce qu'elles couvrent un grand territoire, elles éprouvent de la difficulté à attirer des élèves de l'extérieur des localités où elles sont situées, et ce, en dépit de l'accès au transport scolaire. Par exemple, les parents considèrent que Niagara-Falls est trop éloigné de Welland pour que les jeunes francophones fréquentent l'école secondaire Confédération. De même, à Le Caron, on trouve peu d'élèves de Perkinsfield, de Barrie et de Borden. Même dans les villes où sont situées des écoles de langue française, de jeunes Franco-Ontariens ne les fréquentent pas ou ils prennent la route des établissements de langue anglaise à la fin de leurs études élémentaires. Ainsi, vers 1'an 2000, l'école secondaire Confédération ne recrute environ que $55 \%$ des élèves des écoles élémentaires publiques de langue française de Welland, qui sont supposées constituer ses écoles sources. De même, à l'école élémentaire Saint-Joseph, située à proximité de Le Caron, $40 \%$ des élèves de $8^{\mathrm{e}}$ année ne s'inscrivent pas à ce dernier établissement.

24. Paul-François Sylvestre, Penetang. L'école de la résistance, Sudbury, Prise de Parole, 1980, p. 53-72.

25. Basile Dorion, «Conclusion », dans ibid., p. 73-74. Sur les divisions chez les francophones de la Baie-Georgienne, voir Frenette, «L'Ontario français du Centre et du Sud-Ouest », op. cit., p. 145-183.

26. Cette section sur les problèmes auxquels doivent faire face les écoles secondaires s'appuie sur Lamontagne, Frenette et Bourgeois, loc. cit. 
Dans plusieurs cas, les parents semblent continuer de vouloir que leurs enfants parlent bien l'anglais et ils les envoient dans une école anglophone pour qu'ils acquièrent cette langue. Ces parents proviennent habituellement des couches sociales moins favorisées. Le « complexe du minoritaire » se reflète aussi chez les élèves qui ne fréquentent pas l'école secondaire de langue française ou qui la quittent, de peur qu'elle ne les prépare pas adéquatement au collège ou à l'université de langue anglaise.

La taille réduite des écoles secondaires de langue française fait en sorte qu'elles ne peuvent offrir la même gamme de cours et d'activités parascolaires que les écoles secondaires de langue anglaise. C'est une des raisons qui poussent les élèves à ne pas choisir l'école secondaire de langue française ou à la quitter. Dans les plus petits établissements, les classes à niveaux multiples sont mal perçues par les élèves, qui comparent constamment leur situation à celle de leurs amis dans les établissements de langue anglaise. À Toronto, quelques élèves alternent les semestres et les années scolaires entre Étienne-Brûlé et l'école confessionnelle $\mathrm{M}^{\mathrm{gr}}$ de Charbonnel pour profiter du choix de cours des deux établissements. Partout, des élèves pratiquent l'alternance entre les écoles secondaires de langue française et de langue anglaise. Par ailleurs, pour bien des élèves, il est important d'être fiers de leur école, mais cela s'avère difficile dans un petit établissement où il n'y a pas suffisamment d'activités parascolaires et où les équipes sportives sont peu compétitives en raison du bassin réduit d'athlètes. La coexistence sur le territoire de deux systèmes scolaires, confessionnel et public, et la concurrence parfois acharnée à laquelle ils se livrent, ajoute encore au problème de la petite taille des écoles secondaires.

Si la situation du palier secondaire de langue française dans le Centre et le Sud-Ouest est plutôt précaire dans les trente dernières années du $\mathrm{XX}^{\mathrm{e}}$ siècle, elle devient catastrophique au postsecondaire. Ainsi, au milieu de la décennie de 1990, pas plus de la moitié des 300 finissants du secondaire des deux régions, qui poursuivent des études universitaires en Ontario, ne choisissent 
l'une des trois universités bilingues de la province ${ }^{27}$.

Pour sa part, le collège universitaire Glendon de l'Université York, fondé en 1966, est incapable d'établir une relation durable avec les communautés francophones du Centre et du Sud-Ouest, en raison des caractéristiques mêmes de la population francoontarienne des deux régions (communautés éparpillées sur un grand territoire et plus ou moins coupées les unes des autres, identité collective plus fragmentée qu'ailleurs dans la province, forts transferts linguistiques vers l'anglais), ainsi que de la nature et de la structure même de Glendon. Les diverses tentatives de rapprochement connaissent toutes un succès mitigé. L'institution n'a son premier principal bilingue qu'en 1976 et son premier principal francophone qu'en 1980. De plus, collège d'arts libéraux créé sur le modèle des petites universités élitistes américaines, Glendon n'offre pas de programmes jugés pertinents par la population estudiantine de langue française du Centre et du Sud-Ouest. Ses premiers professeurs, des unilingues anglophones pour la plupart, y sont attirés par sa philosophie des arts libéraux et non pas par son caractère bilingue, qu'ils considèrent comme un mal nécessaire ; au fil des ans, ils embauchent des recrues qui leur ressemblent. Encore au milieu des années 1990, seulement la moitié des 92 professeurs à temps plein peuvent techniquement enseigner en français. En outre, la présence de plusieurs Franco-Européens, peu sensibles à la « question franco-ontarienne », contribue sans doute à aliéner davantage l'institution des communautés de langue française ${ }^{28}$.

Après son arrivée comme principal en 1980, Philippe Garigue obtient des octrois de démarrage et de développement du Conseil de l'éducation franco-ontarienne pour la mise en place de programmes et de cours en langue française destinés en priorité

27. Yves Frenette, « Rapport d'étape du sous-comité sur l'éducation de langue française », Collège universitaire Glendon, 31 janvier 1996.

28. Ce passage sur Glendon provient d'Yves Frenette, « Le Rendez-vous manqué. Le Collège universitaire Glendon et les communautés francophones du centresud-ouest de l'Ontario, 1965-2000», Communication dans le cadre du colloque « Les Francophonies canadiennes minoritaires à l'aube du XXI ${ }^{\mathrm{e}}$ siècle », ACFAS, Université de Montréal, mai 2000. 
aux Franco-Ontariens. Administrateur universitaire chevronné, Garigue comprend l'obligation attachée à ces octrois, mais pas le corps professoral et probablement pas les autorités de l'Université York. D'ailleurs, il appuie son action sur l'idée de l'avènement de Toronto comme grande métropole multiculturelle de moins en moins dominée par les élites anglo-saxonnes, une tendance qui, selon lui, favoriserait le français, non seulement comme langue porteuse de l'identité franco-ontarienne, mais comme langue de communication internationale. Garigue croit que l'avenir du français en Ontario dépend des Franco-Torontois et des anglophones bilingues ; les Franco-Ontariens de l'extérieur de Toronto s'intègrent mal dans la vision du principal de Glendon, puisqu'il les considère comme porteurs de valeurs rurales communautaristes. Le principal tient une série de rencontres avec les associations de langue française du Centre et du Sud-Ouest pour les convaincre du bien-fondé de sa perspective, mais elles ont une tout autre interprétation du rêve de Garigue : les jeunes Franco-Ontariens acquerraient certes de bonnes compétences linguistiques, mais ils demeureraient enracinés dans l'Ontario français. Pour le principal de Glendon, le bilinguisme ne constitue pas « une concession à l'assimilation, mais la reconnaissance des conditions spéciales de la communauté francophone de Toronto, et la situation de notre ville comme métropole commerciale et industrielle ${ }^{29}$ ».

Donc, au début des années 1980, en devenant bénéficiaire d'octrois provinciaux pour les cours et programmes en français, le collège universitaire Glendon est porteur d'un double mandat : desservir la population franco-ontarienne, particulièrement celle du Centre et du Sud-Ouest, tout en permettant à sa clientèle de langue anglaise de poursuivre une instruction bilingue. Pour certains critiques de l'institution, les deux objectifs sont incompatibles. D'ailleurs, les étudiants franco-ontariens ne se manifestent pas ; au nombre d'une demi-douzaine seulement en

29. Cité dans Martin Normand, "Philippe Garigue et l'Ontario français », International Journal of Canadian Studies-Revue internationale d'études canadiennes, $\mathrm{n}^{\text {os }}$ 45-46 (2012), p. 118. 
1980, ils atteignent seulement la trentaine quinze ans plus tard. Heureusement que les étudiants originaires du Québec établissent souvent une résidence légale en Ontario après quelques mois à Toronto.

C'est à ce moment que Dyane Adam devient la première principale franco-ontarienne de 1'histoire de Glendon. Insistant sur la mission sociale du collège, elle lance plusieurs enquêtes auprès des communautés de langue française du Centre et du SudOuest. Attaquée par une partie des membres du corps professoral, qui l'accusent de faire du ethnic cleansing, Adam réussit à faire tomber quelque peu la méfiance des Franco-Ontariens envers Glendon, avant de quitter ses fonctions pour devenir Commissaire aux langues officielles du Canada en 1999.

Au niveau collégial proprement dit, les francophones des deux régions peuvent bénéficier occasionnellement de cours et, encore plus rarement, de programmes en français, jusqu'à la fondation du collège des Grands-Lacs en 1995. Aux prises avec de nombreuses difficultés, l'institution déménage son siège social de Welland à Toronto cinq ans plus tard. Elle offre alors 22 programmes $^{30}$.

La complétude institutionnelle exige aussi que les francophones du Centre et du Sud-Ouest aient accès à des médias de langue française. En 1969, la station CJBC rejoint quelque 85000 auditeurs dans le Centre, mais, dans le Sud-Ouest, il n'existe pas de radio de langue française ${ }^{31}$. Dans les deux régions, l'avènement éventuel de la télévision française suscite de grands espoirs ${ }^{32}$. Elle arrive finalement en 1971 à Toronto et en 1977 à Windsor. À cette date, la radio et la télévision française de Radio-Canada atteignent la plupart des îlots de langue française des deux régions. CJBC rejoint quotidiennement 59000 auditeurs, dont 20000 à l'heure de pointe. Quant à la télévision, elle compte 72000 spectateurs en 1977. Une feuille d'information, Le Rempart, paraît à Windsor à toutes les deux semaines. Le Goût de vivre, journal

30. Grands Lacs. Votre accès à l'emploi. Admission 2000, Toronto, 2000, 33 p.

31. Comité franco-ontarien, op. cit., p. 108-111.

32. André Lapierre, L'Ontario français du Sud-Ouest. Témoignages oraux, Ottawa, Éditions de l'Université d'Ottawa, 1982, p. 157. 
mensuel, s'adresse à la population de la région de LafontainePénétanguishene, tandis que le Hamilton-Express, bimensuel dont le contenu diffère peu de celui du Toronto-Express, fondé en 1973, voit le jour en novembre $1976^{33}$. Publié à Burlington, le bulletin Bonjour est distribué dans le secteur d'Halton et dans le secteur d'Hamilton-Wentworth. Il compte aussi des abonnés à Saint-Catharines, Niagara-Falls et Welland, pour un tirage de $3000^{34}$.

\section{IV - WellaNd : LE dÉClin D'UNe COMMUNAUTÉ FRANCO- ONTARIENNE « TRADITIONNELLE 》}

Jusqu'en 1970, la population de langue maternelle française de Welland avait connu une croissance démographique fulgurante, passant de 931 en 1931 à 7590 en 1971, représentant à cette dernière date $17 \%$ de la population totale de la petite ville industrielle de la péninsule du Niagara. Là comme ailleurs, les Franco-Ontariens s'étaient dotés d'un réseau institutionnel dynamique centré autour de la paroisse. Toutefois, devant l'hostilité des commissaires du système scolaire confessionnel, ils s'étaient tournés vers le conseil scolaire public pour mettre sur pied des écoles élémentaires bilingues. Et, en 1968, ils avaient fait bon usage des lois 140 et 141 en transformant l'école secondaire Sacré-Cœur, ouverte un an plus tôt, en école de langue française publique qui prit le nom d'école secondaire Confédération ${ }^{35}$.

Vers 1970, les Franco-Ontariens constituent une population ouvrière qui est toujours concentrée dans le « quartier social» ou Frenchtown, situé dans l'est de l'agglomération, à proximité des industries qui servent de poumon économique à Welland. « On a

33. Stéphan Larose et Greg M. Nielsen, « Médias et altérité. L'espace public et l'Ontario français virtuel », dans Jacques Cotnam, Yves Frenette et Agnès Whitfield (dir.), La Francophonie ontarienne. Bilan et perspectives de recherche, Hearst, Le Nordir, 1995, p. 303.

34. «Louis Fournier », dans Chamberland, op. cit., p. 329-330.

35. Pour un survol de l'histoire des Franco-Ontariens de Welland, consulter Claude Trudel, Welland, Ottawa, Centre franco-ontarien de ressources pédagogiques, 1982, 148 p. ; Mary-Elizabeth Aubé et Yves Frenette, Communauté française de Welland, Toronto, Fiducie du patrimoine ontarien, 2009, s.p. 
même l'impression », écrivent trois chercheurs, « que ce quartier était l'arrière-cour de l'usine Wabasso ». Avec l'église SacréCœur, le presbytère, la caisse populaire, les commerces, la rue Empire est la « Main Street » de la communauté. Cependant, les francophones, notamment les hommes d'affaires et les membres des professions libérales, ont commencé à s'éparpiller dans de nouveaux quartiers plus cosmopolites ${ }^{36}$.

\section{Tableau 4}

La population de langue française de Welland, 1971-2001

\begin{tabular}{|l|c|c|c|c|c|}
\hline Année & $\begin{array}{c}\text { Population } \\
\text { totale }\end{array}$ & $\begin{array}{c}\text { Langue } \\
\text { maternelle } \\
\text { française }\end{array}$ & $\%$ & $\begin{array}{c}\text { Langue } \\
\text { française parlée } \\
\text { à la maison }\end{array}$ & $\begin{array}{c}\text { Taux de } \\
\text { conservation } \\
\text { linguistique \% }\end{array}$ \\
\hline 1971 & 44395 & 7590 & 17 & 5900 & 77,7 \\
\hline 1981 & 45448 & 7070 & 15,5 & 4645 & 65,7 \\
\hline 1991 & 47914 & 6070 & 12,6 & & 44,0 \\
\hline 2001 & 49895 & 5700 & 11,4 & 2605 & \\
\hline
\end{tabular}

Ce n'est pas là le seul changement. Pour la première fois de son histoire, la communauté franco-ontarienne est privée de sang neuf, le mouvement migratoire en provenance du Québec, de l'Acadie et du nord de l'Ontario se tarissant. Le taux de conservation linguistique baisse de $12 \%$ et le nombre de francophones décline légèrement pendant la décennie, alors que la population de la ville augmente légèrement, ce qui a pour effet de faire passer le pourcentage des francophones dans la population totale à 15,5\% en 1981. Il est révélateur qu'entre 1973 et 1975, les mariages exogames l'emportent pour la première fois sur les mariages endogames dans la paroisse Sacré-Cœur. Même les parents de langue maternelle française n'utilisent pas toujours le français entre eux, surtout parmi les plus jeunes. Par contre, ils parlent le plus souvent à leurs enfants dans cette langue. Pour leur part, les élèves des écoles élémentaires de langue française dont les parents s'expriment surtout en anglais, ont tendance à parler à leurs parents dans cette langue et à préférer les livres anglais aux

36. Joseph-Yvon Thériault, Linda Cardinal et Jean Lapointe, La Communauté francophone de Welland, Ottawa, s.é., 1988, p. 12. 
livres français, et ce dès la deuxième année ${ }^{37}$.

À Welland, $69 \%$ des individus actifs sur le marché du travail œuvrent dans le secteur industriel, où l'anglais domine, en dépit du fait que les Franco-Ontariens représentent parfois entre le cinquième et le quart de la force de travail. Parmi les grandes usines, une seule accorde un statut particulier au français en ayant une politique d'affichage trilingue (français, anglais et hongrois). Il faut dire que $40 \%$ des employés de cette entreprise sont francophones. En fait, seuls les enseignants et les employés de la caisse populaire peuvent gagner leur vie en français.

Les jeunes adultes, c'est-à-dire la tranche d'âge des 20-35 ans, écoutent rarement la radio et la télévision en français ; ils lisent très peu les journaux de langue française ; ils ne trouvent pas les médias francophones attrayants, ne se reconnaissent pas dans la variété de français diffusée sur les ondes. Le même phénomène a cours chez les adolescents, pour qui les organisations socioculturelles de langue française, dont le nombre est quand même appréciable, ne font pas partie du paysage culturel. Le seul club fréquenté en masse par les jeunes, le Club des activités sportives, fonctionne presque totalement en anglais. Enfin, la population de Welland utilise peu les services en français disponibles dans les bureaux du gouvernement fédéral et à la Banque canadienne nationale; même situation dans les commerces de langue française qui desservent aussi une clientèle de langue anglaise.

Toujours dans les années 1970, la grande majorité des élèves du palier élémentaire parlent anglais entre eux après la quatrième année. Cette tendance augmente à l'école secondaire Confédération où certains élèves essaient même d'utiliser cette langue avec leurs enseignants. Le directeur de l'établissement tolère cette

37. L'analyse de la situation linguistique à Welland dans les années 1970 s'appuie sur les nombreuses enquêtes de Raymond Mougeon. Voir notamment Raymond Mougeon, «Le Maintien du français en Ontario », dans Identité culturelle et francophonie dans les Amériques III, Québec, Centre international de recherche sur le bilinguisme, 1980, p. 41-50 ; Raymond Mougeon et Édouard Beniak, « Language Contraction and Linguistic Change. The Case of Welland French », dans Investigating Obsolescence. Studies in Language Contraction and Death, Cambridge, Cambridge University Press, 1989, p. 287-312. 
situation, car il ne veut pas que les élèves soient faibles en anglais, puisqu'ils vont continuer à vivre en Ontario. En général, les jeunes doutent fortement de la valeur du français comme langue de communication. De fait, un bilinguisme anglo-dominant constitue la règle plutôt que l'exception au sein de la jeunesse, un phénomène qui s'observe notamment dans la simplification de la langue française et les emprunts lexicaux à l'anglais.

Quant à l'école secondaire Confédération, elle continue d'être bilingue, le tiers seulement des matières étant offert uniquement en français. C'est que, comme dans d'autres communautés franco-ontariennes à cette époque, les francophones de Welland sont divisés sur la question de la langue d'enseignement au palier secondaire. Pour une partie de la population, composée surtout de membres des professions libérales, d'enseignants et d'autres personnes liées par leur occupation aux institutions de langue française, il est essentiel que les études secondaires se fassent uniquement en français, pour contrer l'assimilation linguistique chez les adolescents. Mais, pour presque la moitié des individus (46,5\%), majoritairement des entrepreneurs, des commerçants et des ouvriers, les jeunes doivent être compétents en anglais s'ils veulent être mobiles socialement, au contraire de leurs parents qui étaient souvent unilingues lors de leur arrivée en Ontario ; $59 \%$ favorisent le bilinguisme. L'école secondaire bilingue représente donc un compromis entre les opinions des deux groupes.

La même division existe au sein du personnel clérical de la paroisse Sacré-Cœur, c'est-à-dire le curé et les trois vicaires, qui desservent une population dont les quatre cinquièmes assistent régulièrement à la messe. Depuis au moins une décennie, l'accroissement des mariages exogames constitue un défi pour eux, les couples dans cette situation demandant des cérémonies religieuses bilingues ou uniquement en anglais. Pour répondre à leurs besoins, la paroisse a recruté en 1964 un vicaire francoaméricain à Lewiston, au Maine. Pour ce dernier, la survie de la paroisse de langue française dépend de sa capacité et de sa volonté d'offrir des services en anglais, faute de quoi les familles 
mixtes se mettront à fréquenter des paroisses de langue anglaise. Selon son estimation, cette politique rapporte, puisque la moitié des foyers mixtes demeure ainsi dans le giron de Sacré-Cœur. Ce à quoi l'un de ses confrères rétorque que la paroisse abandonne ainsi son rôle de défenseur de la langue et de la culture françaises. Raymond Mougeon et Édouard Beniak en concluent :

It [1'Église catholique] can no longer afford to militate actively and overtly in favor of French as it once did, for fear of turning away its (anglicized) faithful. Religion and language, once inseparable, have had to be dissociated, a common evolution within linguistic minorities in North America. ${ }^{38}$

Après 1980, les transformations de la population francoontarienne de Welland s'intensifient, et ce sous plusieurs aspects. D'abord, sa base socio-économique disparaît progressivement. En effet, au gré d'une série de récessions et de la restructuration de l'économie ontarienne, les grands employeurs industriels de la localité disparaissent l'un après l'autre, ce qui entraîne des milliers de mises à pied et une augmentation marquée du taux de chômage ${ }^{39}$. Un recyclage a lieu dans le secteur tertiaire, notamment les centres d'appel et les entreprises de technologie de l'information. En effet, puisque ces employeurs traitent avec des clients de partout au Canada, voire en Amérique du Nord, une main-d'œuvre bilingue ou multilingue est nécessaire pour combler les postes. Ceux-ci représentent une bouée de sauvetage pour les Franco-Ontariens, mais ils n'ont nullement les besoins en main-d'œuvre des industries traditionnelles ${ }^{40}$.

Parallèlement, le « quartier social », qui regroupe encore les trois quarts des francophones en 1980, disparaît progressivement, à la mesure de la mobilité vers les banlieues, les localités voisines ou des cieux extrarégionaux plus cléments. Comme plusieurs institutions, notamment l'église et la caisse populaire, y sont toujours situées, Frenchtown demeure pour les plus vieux

38. Mougeon et Beniak, op. cit., p. 288-289.

39. Thériault, Cardinal et Lapointe, op. cit., p. 7-8.

40. Sylvie Roy, « Le Bilinguisme, les jeunes et le milieu du travail. Maintien d'une communauté ? », Francophonies d'Amérique, vol. 12 (2001), p. 51-59. 
un lieu d'identification important, alors que pour les plus jeunes, il devient lieu de mémoire. Ayant perdu sa base géographique, la communauté franco-ontarienne se déploie plus que jamais dans le secteur associatif : Club Richelieu, Chevaliers de Colomb, Club social, Club sportif, etc. Comme ailleurs, un petit nombre de personnes, une vingtaine peut-être, sont impliquées dans toutes les associations, se remplaçant mutuellement aux conseils d'administration. Par l'entremise de l'AcFo, ces leaders sont bien intégrés à la francophonie régionale et provinciale, au sein de laquelle ils occupent régulièrement des postes importants ${ }^{41}$. L'établissement du siège social du collège des Grands-Lacs à Welland en 1995 constitue un témoignage éloquent de leur poids politique.

Sur le plan scolaire et administratif, on assiste dans les deux dernières décennies $\mathrm{du} \mathrm{Xx}^{\mathrm{e}}$ siècle à une expansion des services en français. Ainsi, l'école secondaire Confédération francise son programme au début de la décennie de 1980, et un deuxième établissement secondaire de langue française, confessionnel celuilà, voit le jour. Avant la mise en œuvre de la Loi 8 en 1988, les services gouvernementaux provinciaux en français sont officiellement inexistants à Welland, à l'exception de ceux du secteur parapublic, où on trouve des foyers pour personnes âgées et une clinique médicale. Toutefois, certains bureaux offrent de façon officieuse des services en français, au gré de la disponibilité d'employés bilingues ${ }^{42}$. La situation s'améliore considérablement après la mise en application de la loi.

Mais, si on se fie aux données du tableau 4, on en conclut que ces gains ont eu peu d'impact sur le fait français. En effet, en 2001, les locuteurs du français ne représentent plus que $11,4 \%$ de la population de la localité; ils sont maintenant 5700 , une baisse de $25 \%$ en trente ans. Le taux de conservation linguistique a aussi périclité : ce n'est plus qu'une minorité de Franco-Ontariens, soit $44 \%$, qui parle principalement le français à la maison. Certains

41. Thériault, Cardinal et Lapointe, op. cit., p. 44-53.

42. Ibid., p. 42-44. 
chercheurs, par exemple Normand Labrie et Sylvie Roy, font montre d'optimisme en faisant remarquer que l'expansion du système scolaire de langue française et l'existence d'employeurs à la recherche de personnel bilingue se combinent pour augmenter la valeur symbolique du français ${ }^{43}$. C'est peut-être vrai, mais une telle tendance ne se manifeste pas dans les statistiques. Au contraire. Au début de la décennie de 1990, Jürgen Erfurt est retourné à l'école Confédération pour répéter l'enquête que Raymond Mougeon avait effectuée en 1978 (tableaux 5 à 11). Les résultats pointent tous dans la même direction : une anglicisation galopante ${ }^{44}$.

\section{Tableau 5}

La langue parlée entre le père et la mère des élèves de l'école secondaire Confédération à Welland, 1978 et 1994 (\%)

\begin{tabular}{|l|c|c|}
\hline & 1978 & 1994 \\
\hline Toujours en français & 52,1 & 16,2 \\
\hline Souvent en français & 13,9 & 10,8 \\
\hline Moitié en français & 6,1 & 11,5 \\
\hline Souvent en anglais & 4,8 & 10,1 \\
\hline Toujours en anglais & 21,2 & 50,7 \\
\hline
\end{tabular}

\section{Tableau 6}

La langue parlée entre les élèves de l'école secondaire Confédération à Welland et leurs pères, 1978 et 1994 (\%)

\begin{tabular}{|l|c|c|}
\hline & 1978 & 1994 \\
\hline Toujours en français & 21,8 & 9,3 \\
\hline Souvent en français & 19,4 & 9,9 \\
\hline Moitié en français & 21,2 & 13,9 \\
\hline Souvent en anglais & 12,7 & 21,2 \\
\hline Toujours en anglais & 24,2 & 45,7 \\
\hline
\end{tabular}

Fait intéressant, la perception de l'importance du français par

43. Labrie, op. cit.; Roy, loc. cit.

44. Jürgen Erfurt, «Le Changement de l'identité linguistique chez les FrancoOntariens. Résultats d'une étude de cas », dans Normand Labrie et Gilles Forlot (dir.), L'Enjeu de la langue en Ontario français, Sudbury, Prise de Parole, 1999, p. 59-77. Les tableaux 5 à 11 proviennent de cette étude. 
Tableau 7

La langue parlée entre les élèves de l'école secondaire Confédération à Welland et leurs mères, 1978 et 1994 (\%)

\begin{tabular}{|l|c|c|}
\hline & 1978 & 1994 \\
\hline Toujours en français & 26,5 & 8,6 \\
\hline Souvent en français & 16,5 & 11,8 \\
\hline Moitié en français & 22,4 & 23,7 \\
\hline Souvent en anglais & 19,4 & 29,6 \\
\hline Toujours en anglais & 14,1 & 26,3 \\
\hline
\end{tabular}

Tableau 8

La langue parlée entre les élèves de l'école secondaire Confédération à Welland et leurs frères et sœurs, 1978 et $1994(\%)$

\begin{tabular}{|l|c|c|}
\hline & 1978 & 1994 \\
\hline Toujours en français & 3,6 & 1,4 \\
\hline Souvent en français & 6,7 & 5,1 \\
\hline Moitié en français & 22,4 & 11,6 \\
\hline Souvent en anglais & 30,3 & 37,7 \\
\hline Toujours en anglais & 37,0 & 44,2 \\
\hline
\end{tabular}

Tableau 9

La langue parlée à la maison entre les élèves de l'école secondaire Confédération à Welland et leurs amis, 1978 et 1994 (\%)

\begin{tabular}{|l|r|r|}
\hline & 1978 & 1994 \\
\hline Toujours en français & 1,2 & 0,0 \\
\hline Souvent en français & 2,9 & 3,3 \\
\hline Moitié en français & 13,5 & 7,2 \\
\hline Souvent en anglais & 26,3 & 30,9 \\
\hline Toujours en anglais & 55,6 & 68,6 \\
\hline
\end{tabular}

les élèves a peu évolué (tableau 10). Connaître le français dans un pays officiellement bilingue confère à ses locuteurs un prestige nouveau. C'est sans doute la raison principale pour choisir de poursuivre ses études secondaires en français. Parfois, les jeunes expriment leur motivation par leur attachement à la langue française, mais, le plus souvent, c'est la valeur ajoutée du bilinguisme qui les amène à l'école secondaire Confédération, même ceux qui 
proviennent de foyers homogènes francophones.

Tableau 10

La perception de l'importance du français chez les élèves de l'école secondaire Confédération à Welland, 1978 et 1994 (\%)

\begin{tabular}{|l|r|r|}
\hline & 1978 & 1994 \\
\hline Très important & 28,1 & 20,9 \\
\hline Important & 38,0 & 45,8 \\
\hline Assez important & 30,4 & 26,8 \\
\hline Peu important & 3,5 & 5,9 \\
\hline Pas important & 0,0 & 0,7 \\
\hline
\end{tabular}

V - TORONTO : L'AVÈNEMENT D'UNE NOUVELLE FRANCOPHONIE À l'aube du XXI ${ }^{\mathrm{e}}$ siècle, la communauté franco-torontoise, qui compte 57410 personnes représentant 1,2\% de la population de la ville, possède un réseau institutionnel qui n'a rien à envier aux autres centres de population de langue française du Centre et du Sud-Ouest : deux paroisses; sept garderies; une colonie de vacances ; onze écoles élémentaires et trois écoles secondaires réparties entre le Conseil scolaire de district du Centre-Sud-Ouest et le Conseil scolaire de district catholique du Centre-Sud, en plus d'un lycée français ; le Collège des Grands-Lacs ; le Collège universitaire Glendon ; deux hebdomadaires; une station de radio et deux stations de télévision ; un Salon du livre dont c'est la 8 éédition en l'an 2000 ; une société historique ; un centre médico-social-communautaire ; un centre pour personnes âgées et une maison de soins de longue durée ; un centre de ressources pour immigrants ; une dizaine d'associations et de clubs, dont le prestigieux Cercle canadien ; un théâtre français ; un comité français à l'Hôtel-de-ville. Cette liste n'inclut pas les nombreux services en français et bilingues fournis par les gouvernements fédéral et provincial ainsi que par l'entreprise privée. En effet, des concessionnaires d'automobiles aux agences d'assurance en passant par les boîtes de traduction et de révision, il est possible de trouver des interlocuteurs francophones. En plus, comme dans 
la majorité des grandes villes nord-américaines, la présence de 5000 à 7000 immigrants français est synonyme de créneaux associés à l'Hexagone, comme des restaurants, des traiteurs, des coiffeurs, etc. ${ }^{45}$

Depuis 1970, le nombre de Franco-Torontois est demeuré plus ou moins stable en chiffres absolus ; contrairement à celle de Welland, la population de langue française n'a pas décliné, mais elle n'a pas fait non plus de grands gains. Dans la Ville reine, les pertes démographiques dues aux transferts linguistiques ont été compensées par l'arrivée constante de francophones venus d'ailleurs. En 2001, moins du tiers (30 \%) des Franco-Ontariens de Toronto sont nés en Ontario ; $47 \%$ proviennent d'une autre province canadienne et $23 \%$ de l'étranger. Le tiers des francophones sont membres d'une minorité raciale.

Une étude réalisée par la sociologue Diane Gérin-Lajoie entre 1997 et 2000 auprès de 89 élèves du seul établissement secondaire confessionnel de langue française de Toronto, $\mathrm{M}^{\mathrm{gr}}$ de Charbonnel, confirme la diversité ethnoculturelle de la francophonie torontoise à la fin $\mathrm{du} \mathrm{Xx}^{\mathrm{e}}$ siècle (tableau 11). Presque la moitié des élèves sont nés dans la métropole ou dans sa région immédiate, mais à peine $6 \%$ de leurs mères et $7 \%$ de leurs pères en sont originaires. Et si le pourcentage des Franco-Ontariens de souche parmi les élèves atteint presque $60 \%$, il est respectivement de $17 \%$ chez les mères et de $16 \%$ chez les pères. En fait, le cinquième des élèves, presque la moitié des mères et $60 \%$ des pères proviennent de l'extérieur du Canada. La proportion de Québécois dans les trois groupes est respectivement de $17 \%, 26 \%$ et $16 \%$. À l'opposé, les 23 membres du personnel enseignant de l'école sont surtout franco-ontariens « de souche », bien que quelques-uns d'entre eux soient originaires du Québec, de l'Acadie, de l'Espagne et du Liban ${ }^{46}$.

Source : Diane Gérin-Lajoie, Parcours identitaires de jeunes francophones en milieu minoritaire, Sudbury, Prise de Parole, 2003, p. 43, 46.

45. Annuaire des ressources francophones de Toronto 2000, Toronto, Centre francophone du Toronto métropolitain, 2000, $55 \mathrm{p}$.

46. Diane Gérin-Lajoie, Parcours identitaires de jeunes francophones en milieu minoritaire, Sudbury, Prise de Parole, 2003, p. 34-46. 
Tableau 11

Lieux de naissance des élèves et des parents de l'école $M^{\text {gr }}$ de Charbonnel

\begin{tabular}{|l|c|c|c|}
\hline & Élèves $\%$ & Mères \% & Pères \% \\
\hline Ontario indéterminé & 2,9 & 2,9 & 0,0 \\
\hline Ontario Nord & 0,0 & 4,3 & 4,3 \\
\hline Ontario Sud-Ouest & 2,9 & 2,9 & 2,9 \\
\hline Ontario Centre & 48,0 & 5,7 & 7,2 \\
\hline Ontario Est & 4,3 & 1,4 & 1,4 \\
\hline Québec & 17,3 & 26,1 & 16,0 \\
\hline Nouveau-Brunswick & 0,0 & 1,4 & 1,4 \\
\hline Ailleurs Canada & 4,3 & 2,9 & 4,3 \\
\hline Ailleurs monde & 20,2 & 49,2 & 59,3 \\
\hline Sans réponse & 0,0 & 2,9 & 2,9 \\
\hline Total & 99,9 & 99,7 & 99,7 \\
\hline
\end{tabular}

Quant à la langue des parents, les mères sont de langue maternelle française à $46 \%$ et de langue maternelle anglaise à $15 \%$; chez les pères, les pourcentages sont respectivement de $39 \%$ et de $22 \%$. Les proportions d'allophones se ressemblent dans les deux sexes, soit $38 \%$ chez les femmes, $36 \%$ chez les hommes ${ }^{47}$.

En ce tournant de siècle, la francophonie torontoise est donc très diversifiée. Elle est aussi très éparpillée. Les principales concentrations de francophones se retrouvent sur le Waterfront, dans le corridor University-Parliament, de part et d'autre de la rue Yonge au sud de Bloor, ainsi qu'un peu plus au nord dans le même axe, autour de l'intersection Yonge-Eglinton, ne formant nulle part une forte proportion de la population, rarement plus de $5 \%$, ce qui contribue à leur invisibilité ${ }^{48}$. Par ailleurs, les Franco-Canadiens de souche vivent davantage dans les banlieues éloignées, alors que les immigrants de langue française habitent le centre-ville et les banlieues rapprochées. Comme l'écrivent les géographes Anne Gilbert et André Langlois,

La situation centrale de la population intégrée à la francophonie torontoise a certes ses avantages. Elle se trouve ainsi là où la

47. Ibid., p. 47.

48. Anne Gilbert et André Langlois, « Organisation spatiale et vitalité des communautés francophones des métropoles à forte dominance anglaise du Canada », Francophonies d'Amérique, no 21 (2006), p. 111-114. 
taille de la communauté francophone mais aussi son poids sont les plus forts. Elle se concentre aussi dans les quartiers où l'on retrouve une plus grande densité institutionnelle francophone à l'échelle métropolitaine, notamment en ce qui concerne l'enseignement postsecondaire et la santé [...] Non seulement la francophonie issue de l'immigration peut-elle profiter de ces services, mais elle peut aussi contribuer à les développer, par une participation active aux structures qui sont en place. Toutefois, le fait que la francophonie de souche et la francophonie issue de l'immigration occupent des milieux différents au sein de l'espace métropolitain représente un défi sérieux pour l'intégration des nouveaux arrivants à la communauté francophone ${ }^{49}$.

Invisibilité, diversité, éparpillement géographique, coexistence d'enclaves, tensions et conflits ethnoculturels ne sont pas des caractéristiques nouvelles de la population de langue française de la Ville reine, comme nous l'avons vu ailleurs ${ }^{50}$. En 1971, 13,2 \% des francophones étaient nés à l'extérieur du Canada, la majorité en France. Pour des raisons qu'il est difficile à saisir, le conflit qui avait éclaté au grand jour en 1968 entre Français et Canadiens français au sujet de la fondation d'une Maison française se résorba. Si certains immigrants français venus à Toronto pour apprendre l'anglais et découvrir le monde anglo-saxon évitaient de s'associer avec d'autres francophones, certains de leurs compatriotes travaillaient à des projets communs avec les Franco-Ontariens « de souche » et les Québécois ${ }^{51}$. Pour sa part, le Consulat de France, de concert avec des organismes français, jouait un rôle important au sein de la francophonie torontoise, par exemple en participant à l'organisation annuelle d'un salon du livre à partir de 1993 et l'établissement d'une succursale de la Librairie Renaud-Bray en

49. Id., " Mesure et géographie de la francophonie torontoise », dans Anne Gilbert (dir.), Territoires francophones. Études géographiques sur la vitalité des communautés francophones du Canada, Québec, Septentrion, 2010, p. 88.

50. Frenette, "L'Ontario français du Centre et du Sud-Ouest », op. cit., p. $145-183$.

51. Gilles Forlot, «Minorité et légitimité communautaire. La migration française de Toronto entre francophonie et anglophonie ", Francophonies d'Amérique, $\mathrm{n}^{\circ} 21$ (2006), p. 134-136. 
199552. La même année, soucieux d'implanter des institutions franco-françaises, il suscita également l'ouverture d'un lycée françai ${ }^{53}$. La présence du consulat contribuait au prestige des immigrants français.

Par contre, dans les décennies de 1970 et de 1980 continuaient de coexister deux communautés francophones distantes, celle de la banlieue et celle du centre-ville. La première était composée presqu'entièrement de Québécois nouvellement arrivés et d'une poignée de familles franco-ontariennes du nord et de l'est de la province. Pour sa part. la communauté du centre-ville était constituée d'éléments plus disparates : Franco-Ontariens, Québécois et Acadiens ; francophones d'Europe, du Moyen-Orient, de l'Afrique du Nord et d'Haïti. Le point de regroupement des francophones de la banlieue était le club social et culturel, dont ils se dotèrent sans doute pour faciliter la transition entre leur milieu d'origine et leur nouveau train de vie. Très actif, le club social offrait un nombre impressionnant d'activités : pique-niques, sports, jeannettes et guides, louveteaux et castors, théâtre amateur, carnaval d'hiver, camping familial, fêtes de Noël pour les enfants, danses, rallye automobile, tournois, parties de cartes, spectacles. De plus, certains membres du club se regroupèrent pour fonder une école élémentaire confessionnelle de langue française, puis pour exercer des pressions politiques, quand il fut question de déménager ladite école. D'autres firent les démarches nécessaires à la création d'une paroisse, prenant toutes les initiatives auprès de l'Archevêché de Toronto. L'organisation de la nouvelle paroisse dans ses menus détails fut l'œuvre des francophones de la banlieue $^{54}$.

La communauté de langue française du centre-ville différait

52. Cette initiative fit long feu, la succursale fermant ses portes dès 1996.

53. La Lettre $d u$ Consulat général de France, $\mathrm{n}^{\circ} 8$ (décembre, janvier, février 1994-1995), p. 4-5; nº 10 (juin-octobre 1995), p. 4 ; n 11 (novembre-janvier 1995-1996), p. 1-5.

54. Cette section sur les deux communautés francophones de Toronto s'inspire de Normand Frenette, «Franco-Torontois et Franco-Ontarien. Cheminement individuel et collectif », Revue de l'Université d'Ottawa, vol. 15, nº 2 (1985), p. 154-155. 
beaucoup. Même si elle comprenait de nombreux clubs sociaux, voire une fédération de ces clubs, ceux-ci ne jouaient pas un rôle aussi important dans les loisirs des francophones que dans la banlieue. Les individus fréquentaient les différents organismes beaucoup plus en consommateurs, utilisant leurs services selon les besoins du moment et du projet en cause. En d'autres termes, la communauté du centre-ville se faisait et se défaisait au gré des projets de l'heure. C'était en partie parce que les francophones ne partageaient pas une mémoire et un vécu.

Partout en Ontario, la renaissance de la question scolaire suscita au tournant des années 1970 des tensions et des conflits entre francophones et anglophones, lorsque les premiers revendiquèrent leurs droits à une instruction en français, notamment au palier secondaire, comme ce fut le cas dans Kent-Essex et à la Baie-Georgienne. À cet endroit et à Welland, les Franco-Ontariens étaient divisés quant à la place du français dans leurs écoles secondaires. Toronto représentait un cas de figure unique, puisque les débats, qui se transformaient souvent en luttes, portaient sur le rôle de l'école de langue française dans la reproduction culturelle et la place des anglophones à l'intérieur du système scolaire francophone. En effet, dans la Ville reine, l'avènement du bilinguisme canadien et un sentiment de bonne entente suscitèrent chez des anglophones de la classe moyenne un véritable engouement pour le fait français. En pratique, cela se traduisit par le mouvement de l'immersion d'élèves anglophones dans des classes spéciales où l'enseignement était dispensé surtout ou complètement en français. Les premières classes d'immersion furent mises sur pied aux écoles Allenby en 1971 et Brown en 1972. Cinq ans plus tard, le mouvement prit de l'ampleur lorsque les autorités provinciales firent connaître leur désir d'encourager l'enseignement du français. Nombre de parents demandèrent alors aux conseils scolaires public et confessionnel de mettre sur pied des classes d'immersion. Entre 1979 et 1985, le nombre d'élèves en immersion passa de 989 à 3600 ; ils représentaient alors 
$6,5 \%$ des élèves de Toronto ${ }^{55}$.

Toutefois, certains francophiles ne croyaient pas que les programmes d'immersion réussiraient à former des individus vraiment bilingues. Ils s'impliquèrent plutôt dans la fondation d'établissements scolaires de langue française. Ainsi, entre 1972 et 1975, le journaliste Clay Derstine prit la tête d'un mouvement pour créer la première école élémentaire publique de langue française en Ontario, Gabrielle-Roy. En 1978, Derstine joua également un rôle clef dans la fondation du module français du Collège Jarvis, une école secondaire publique. Cofondateur de la garderie Les Bouts d'choux en 1982, Derstine fut tout au long de la décennie de 1980 l'homme politique le plus en vue sur la scène scolaire francophone, notamment comme membre du CCLF au Conseil scolaire de Toronto. Opposé à la mise sur pied d'un conseil de langue française autonome pour le Grand-Toronto, il proposa plutôt une nouvelle formule de conseil consultatif de langue française. En dépit de l'action de Derstine et de ses alliés, le Conseil des écoles françaises de la communauté urbaine de Toronto (dorénavant CÉFCUT) vit le jour en 1988 sous l'impulsion de parents francophones; la nouvelle entité regroupait trois écoles élémentaires (Gabrielle-Roy, Jeanne-Lajoie, Félix-Leclerc) et deux écoles secondaires (module de langue française de l'Institut collégial Jarvis, Étienne-Brûlé), qui avaient été auparavant sous la houlette du Conseil scolaire de Toronto ou du Conseil scolaire de North-York. Quant aux écoles confessionnelles de langue française, elles demeuraient sous la juridiction du Conseil des écoles catholiques de Toronto ${ }^{56}$.

Pendant toute son existence, le CÉFCUT constitua un champ de bataille entre le groupe de Derstine, composé surtout de francophiles, et ses opposants, en grande partie des Franco-Canadiens. Les premiers trouvèrent des alliés au sein de l'élite immigrante

55. Michel Genty, «Le Trillium et le lys ou quelques observations sur les classes d'immersion en français, à Toronto et en Ontario ", Études canadiennes - Canadian Studies, no 22 (1987), p. 83-86.

56. Marie-Josée Métivier, « Les francophones de Toronto dirigent enfin leurs écoles », Langue et société, vol. 26 (printemps 1989), p. 31-32. 
de langue française, qui était alors presque entièrement d'origine européenne et nord-africaine. Les seconds étaient appuyés par 1'ACFo régionale. Les ennemis s'affrontaient lors des élections scolaires et, puisqu'ils réussissaient simultanément à faire élire des représentants, la guerre se déplaçait aux réunions du CÉFCUT ${ }^{57}$. Les mêmes combats avaient lieu au sein même des écoles.

L'enjeu était de taille : la nature des établissements scolaires. À ses débuts, en 1975, les quatre cinquièmes de la clientèle de l'école Gabrielle-Roy étaient anglophones, puisque l'établissement était ouvert à tous. Encore dix ans plus tard, le tiers seulement des 545 élèves étaient de langue maternelle française, le reste étant constitué d'élèves de langue anglaise appartenant à 45 nationalités ; il s'agissait surtout d'enfants de cadres et d'intellectuels, dont beaucoup de professeurs de l'Université de Toronto et de 1'Université York ${ }^{58}$. Au module français du Collège Jarvis en 1990, 65 \% des élèves étaient anglophones ${ }^{59}$. La même situation prévalait dans les établissements confessionnels de langue française. À l'école Sacré-Cœur, au centre-ville de Toronto, 11 \% des parents formaient des couples homogènes de langue anglaise, $40 \%$ des couples mixtes et $30 \%$ de couples homogènes de langue française ${ }^{60}$. En tout et pour tout, dans l'ensemble des écoles de langue française des deux systèmes, la moitié des élèves étaient de langue maternelle française ${ }^{61}$.

Pour les tenants de la francisation, les écoles de langue française devaient desservir surtout les familles d' expression française et renforcer la culture franco-ontarienne. Ces parents souhaitaient que le conseil scolaire resserre les critères d'admission de façon rigoureuse pour que l'école devienne vraiment française. Pour

57. Archives personnelles de l'auteur, Carton électoral, 1991.

58. Genty, op. cit., p. 97.

59. Archives personnelles de l'auteur, « Témoignage. Tous des candidats au conseil scolaire public » (Jacinthe Fraser), texte dactylographié, s.d.

60. Monica Heller, « The Role of Language in the Formation of Ethnic Identity ", dans J. Phinney et M. Rotheram (dir.), Children's Ethnic Socialization. Identity, Attitudes and Interactions, Newbury Park, Sage Publications, 1987, p. 190-191.

61. Id., «L'École de langue française à Toronto », Revue du Nouvel-Ontario, vol. 9 (1987), p. 73. 
leurs opposants, on n'avait pas le droit d'exclure les élèves dont les parents ne parlaient pas couramment français, puisque parents et enfants pouvaient apprendre cette langue. En outre, les francophiles évoquaient la nécessité de maintenir un certain niveau d'inscriptions pour pouvoir assurer une gamme de services comparables à ceux des écoles de langue anglaise. Les défenseurs de ce point de vue utilisaient également les arguments de la réussite scolaire des enfants non francophones, du caractère bilingue et biculturel du Canada, et des vertus de l'ouverture d'esprit :

I have two kids who are fluently bilingual in a bilingual country and not only have I no guilt I sort of felt proud of it [...] we made a commitment to the fact that there are two languages and two cultures. That's why Ifeel so strong about anyone questioning whether it's the right or wrong thing - it's perfectly right, it's perfectly moral. ${ }^{62}$

Selon Monica Heller, le premier point de vue était surtout celui des couples de la classe moyenne pour qui la langue française représentait souvent une plus-value dans leur emploi (ou ils envisageaient que ce serait le cas pour leurs enfants); ces parents étaient en général bilingues et ils étaient très conscients du statut minoritaire des Franco-Ontariens. Le deuxième point de vue caractérisait surtout les anglophones qui voulaient conserver leur accès à l'école « authentiquement française »; ces parents affirmaient détenir un droit historique, puisqu'ils avaient joué un rôle majeur dans la création de plusieurs écoles de langue française. N'ayant pas d'expérience en situation minoritaire, ils ne comprenaient pas, pour la plupart, le protectionnisme de certains parents francophones et leur peur de l'assimilation. Entre les deux groupes, on trouvait des familles de la classe ouvrière et des unilingues francophones récemment arrivés qui s'inquiétaient de leur accès au monde anglophone et qui n'étaient pas complètement convaincus de la valeur de leur capital linguistique français dans

62 Ibid, p. 75-77 (citation p. 77). Voir aussi la monographie qu'Heller a consacrée à la question : Crossroads. Language, Education and Ethnicity in French Ontario. Berlin, Mouton de Guyter, 1994, 252 p. 
le domaine du travail ou d'autres secteurs ${ }^{63}$.

C'est le premier groupe qui gagna la bataille. Peu à peu, les francophiles et leurs enfants disparurent de la scène scolaire francophone. De toute façon, dans les années 1990, à l'école comme ailleurs, la question de l'immigration commençait à occuper la place centrale au sein de la francophonie de la Ville reine ${ }^{64}$.

Beaucoup d'immigrants francophones, y compris les réfugiés politiques, étaient passés par un pays intermédiaire ou par le Québec avant d'aboutir à Toronto ${ }^{65}$. À leur arrivée, leur répertoire linguistique s'était donc déjà enrichi des variétés de l'anglais et du français québécois. La présence de membres de leur parenté ou d'un réseau de connaissances était fondamentale dans leur établissement. Après leur arrivée dans la capitale de l'Ontario, le processus de mobilité géographique se poursuivit, du centre-ville vers les banlieues et parfois vers d'autres centres urbains canadiens. Parmi les mieux nantis, certains retournaient temporairement dans leur pays natal pour revoir les membres de leur famille ou pour maximiser les opportunités économiques ${ }^{66}$.

Comme les immigrants anglophones et allophones, les immigrants francophones faisaient face à de multiples obstacles en raison du taux de chômage élevé, du sous-emploi, de leur arrivée récente, de la non-reconnaissance de leurs diplômes et de la discrimination ${ }^{67}$. Les groupes qui avaient commencé à s'établir à Toronto dans les années 1970, les Haïtiens par exemple, disposaient d'assises institutionnelles plus solides que les groupes

63. Id., « L’École de langue française à Toronto », p. 77-78.

64. Pour la problématique de l'immigration dans la francophonie canadienne, consulter Diane Farmer, "L'Immigration francophone en contexte minoritaire. Entre la démographie et l'identité », dans Joseph-Yvon Thériault, Anne Gilbert et Linda Cardinal (dir.), Francophones et minoritaires. Nouveaux enjeux, nouvelles perspectives. Montréal, Fides, 2008, p. 121-159; Yves Frenette, « Immigration et francophonie canadienne au tournant du XXI ${ }^{\mathrm{e}}$ siècle », dans Dean Louder et Éric Waddell (dir.), Franco-Amérique, Québec, Septentrion, 2008, p. 345-355.

65. Pour un exemple, voir « Pierre-Eddy Toussaint », Chamberland, op. cit., p. $442-443$.

66. Adrienne Chambonetal., L'Immigrationet la communautéfranco-torontoise, Toronto, Centre de recherches en éducation franco-ontarienne, 2001, p. 3-5.

67. Ibid., p. 5. 
immigrés plus tard. La fondation de la Confrérie haïtienne du Canada en 1979 fut suivie de celles du Cercle des Égyptiens l'année suivante et de l'Association des Marocains de Toronto en 1981. Quelques années plus tard, soit en 1987-1988, une tentative d'unification des immigrants de langue française eut cours, lorsque l'Association multiculturelle francophone de l'Ontario (dorénavant AmFo) voit le jour. Toutefois, des divergences idéologiques et des conflits de personnalité provoquèrent une scission au sein de l'organisme, ce qui donna naissance en 1989 à l'Association interculturelle franco-ontarienne, qui se différenciait de l'AMFo en prônant un rapprochement avec les organismes franco-ontariens, afin de faire avancer les grands dossiers de la francophonie provinciale, tout en défendant spécifiquement les intérêts et besoins de ses membres ${ }^{68}$.

Dans la dernière décennie $\mathrm{du} \mathrm{xx}^{\mathrm{e}}$ siècle, l'arrivée de plus en plus massive d'immigrants francophones eut un grand impact sur les organismes franco-torontois. En général, ceux-ci avaient été mis sur pied par des militants franco-ontariens « de souche», souvent après d'âpres luttes. Avec l'établissement de nouveaux arrivants de langue française, ces organismes durent remettre en question leur raison d'être et leur fonctionnement. Ainsi, le Franco-Club, né dans les années 1970 du regroupement de divers organismes, s'était voulu à l'origine un centre communautaire et culturel au service des francophones de la Ville reine. Cette vocation s'était développée grâce à l'appui du gouvernement fédéral, intéressé qu'il était à promouvoir sa vision du bilinguisme au cœur de Toronto, et des gouvernements provincial et municipal. Dans les années 1990, le Franco-Club dut se réorienter, les pouvoirs publics voyant dans l'organisme un intermédiaire apte à fournir des services aux nouveaux arrivants. Il se transforma donc progressivement en service d'accueil. Cependant, sa légitimité reposait toujours sur son image de point de ralliement de la communauté francophone. Par ailleurs, ses programmes pour les nouveaux arrivants étaient censés répondre à des exigences

68. L'Express, 3-9 avril 2007. 
administratives établies par les gouvernements ; or il n'y avait pas d'adéquation entre les besoins des immigrants et les normes gouvernementales, ce qui résulta en un certain mécontentement envers les services offerts par le Franco-Club ${ }^{69}$.

Comme nous l'avons vu, les immigrants et les enfants d'immigrants tiennent une place importante dans les écoles de langue française de la Ville reine. Le corps estudiantin est composé d'une population multilingue, aux registres variés, en français et en anglais. Toutefois, pour les conseils scolaires, les directions et les enseignants, l'école demeure envisagée comme un territoire monolingue. Elle favorise une gamme de variétés du français, mais cette gamme se limite aux standards régionaux, surtout ceux définis par la France ou bien celui en voie d'émergence au Canada. Les événements organisés par l'école mettent l'accent sur la mémoire canadienne-française, mais ils révèlent aussi des tensions dans cette expression identitaire, en relativisant cette mémoire par des références à la francophonie internationale et à la présence d'autres formes identitaires. Les élèves bilingues sont ceux qui participent le plus aux événements mettant en avant le côté francophone de l'école, soit par leur travail bénévole, soit lors des spectacles par des applaudissements et une participation physique à la musique. Cette vision bilingue, branchée et moderne, marginalise la tradition canadienne-française en l'affublant d'une image passéiste, bornée et provinciale. Ces élèves construisent une image bilingue de l'école avec des emprunts à l'anglais, un français influencé par l'anglais, une dévalorisation du français québécois, le non-emploi du français européen et des renvois à la culture anglo-américaine. Quant à eux, les élèves d'origine africaine ont leur propre image de la francophonie ; ils utilisent des structures linguistiques afro-canadiennes de langue anglaise et sont tiraillés entre le rejet de l'impérialisme européen et le prestige associé au français européen. En outre, ils ont parfois de

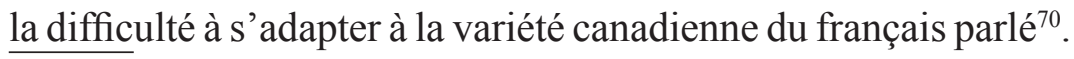

69. Chambon, op. cit., p. 8.

70. Monica Heller, «Quel(s) français et pour qui ? Discours et pratiques identitaires en milieu scolaire franco-ontarien », Labrie, op. cit., 1999, p. 129-165. 


\section{ÉPILOGUE}

Dans les trois dernières décennies du $\mathrm{Xx}^{\mathrm{e}}$ siècle, on peut se demander si, en dépit du fractionnement qui ressort de notre étude, une conscience régionale francophone n'est pas en voie d'éclosion dans le Centre et le Sud-Ouest. D'une part, la primauté économique du secteur tertiaire et le rôle grandissant de l'État provincial amènent à Toronto et ailleurs des Québécois, des Franco-Ontariens d'autres régions, des Acadiens et des francophones de l'extérieur du Canada. La population combinée du Centre et du Sud-Ouest est ainsi devenue la plus instruite de l'Ontario français et celle qui bénéficie du revenu le plus élevé. Avec l'avènement de la loi 8 en 1986, Toronto s'est transformée en métropole francophone régionale. Les institutions et la culture franco-torontoises rayonnent dans tout le Centre et, à un degré moindre toutefois, dans le Sud-Ouest.

Les autres gouvernements jouent également un rôle important dans l'émergence de cette conscience régionale francophone. Comme Queen's Park, l'État fédéral a établi des structures qui facilitent la concertation; la nouvelle ville du Grand-Toronto, créée en 1998, fait de même. Surtout, l'acquisition de la gestion scolaire par les Franco-Ontariens et la restructuration des conseils scolaires contribuent de façon marquée à cette nouvelle conscience régionale. Le Conseil scolaire de district du Centre-Sud-Ouest couvre toute la région, alors que les deux conseils catholiques de langue française recoupent les frontières du diocèse de Toronto, c'est-à-dire le Centre, et de celui de London, essentiellement le Sud-Ouest.

Parallèlement, et peut-être paradoxalement, la caractéristique principale de la nouvelle région franco-ontarienne du CentreSud-Ouest demeure, nous le répétons, son morcellement géographique et son éclatement identitaire. Dans les écoles secondaires de langue française de la région, l'anglais domine largement à l'extérieur de la salle de classe et les élèves ont souvent honte de parler français, notamment dans la rue. Leur relation à la langue française est presque uniquement socio-économique : le bilin- 
guisme va leur procurer un bon emploi. Ces jeunes se définissent comme canadiens bilingues, et non comme franco-ontariens ou francophones. Ils dissocient langue française et culture francoontarienne, cette dernière leur apparaissant ancienne et dépassée.

Quoi qu'il en soit, une chose est certaine : les francophones du Centre-Sud-Ouest représentent le tiers des Franco-Ontariens. Les politiques publiques et les stratégies de développement communautaire devront tenir compte de leurs besoins, en dépit et en raison de leur spécificité. 